\title{
Spectro-chemometric determination of panthenol enantiomeric excess in pharmaceutical products
}

\author{
Roman Poláček ${ }^{1}$, Angela Kleinová ${ }^{\text {, Pavel Májek }}{ }^{3}$ \\ ${ }^{1}$ EUROFINS BEL/NOVAMANN s.r.o., Komjatická 73, 94002 Nové Zámky, Slovak Republic \\ ${ }^{2}$ Polymer institute, Slovak Academy of Sciences, Dúbravská cesta 9, 84541 Bratislava, Slovak Republic \\ ${ }^{3}$ Institute of Analytical Chemistry, Faculty of Chemical and Food Technology, Slovak University of Technology, \\ Radlinského 9, 81237 Bratislava, Slovak Republic \\ pavel.majek@stuba.sk
}

\begin{abstract}
Antibiotic, soothing and healing properties of panthenol are exploited in various pharmaceutical and cosmetic products. Only D-panthenol is biologically active while its L-form might counteract the biological effectiveness of the D-enantiomer. Hydrating and moisturizing effects are exhibited by both enantiomers. Therefore, it is necessary to develop a rapid and cheap method for the determination of panthenol enantiomeric excess in pharmaceutical preparations. In this study, $\beta$-cyclodextrin was used as a chiral selector for the recognition of panthenol enantiomers. Inclusion complexes formed by $\beta$-cyclodextrin and the analyte showed small differences in NIR and UV/VIS spectra compared by chemometric assessment. Based on the figure of merit and model characteristics, PLS calibration model in the selected range of NIR spectra is preferred. UV/VIS spectrometry has the disadvantage of complicated sample preparation compared to NIR spectrometry. Results reached by both proposed methods were in good agreement. Statistical investigation of the results (by Student $t$-test and Fisher $F$-test) confirmed that the proposed methods are comparable and applicable to chiral analysis of panthenol pharmaceutical preparations.
\end{abstract}

Keywords: chiral analysis, panthenol, pharmaceutical preparations, molecular spectrometry, multivariate calibration

\section{Introduction}

D-panthenol (D-(+)-2,4-dihydroxy-N-(3-hydroxypropyl)-3,3-dimethybutylamide) is an alcoholic analogue of pantothenic acid, a member of the B complex vitamins (vitamin B5). It is also known as pantothenyl alcohol or provitamin B5. It is found in various commercial products, e.g. skin creams, hair preparations, eye drops as well as in tablets or injections for its anti-inflammatory and regenerating properties (Ebner et al., 2002). Dexpanthenol is enzymatically oxidized to pantothenic acid, which is a constituent of coenzyme A. The acid form plays an important role in the Krebs cycle and metabolism of lipids, proteins, and hormones. The lack of pantothenic acid causes many kinds of deficiency disease, irritation of skin, dermatitis, depigmentation of hair and stunted growth (Khater et al., 2015).

Only D-panthenol is biologically active while the L-form is at the best biologically inert and might even partly counteract the biological effectiveness of the D-enantiomer. On the other hand, both D- and L-panthenol appear to have hydrating and moisturizing effects on the skin because both enantiomers chemically attract water (König et al., 1985;
Takashi et al., 1989). The above mentioned properties are very important for the enantiomeric excess (EE) determination of panthenol in pharmaceutical preparations. Chiral analysis of panthenol is usually performed by HPLC (Xie et al., 2009; Mao et al., 2010; Hroboňová et al., 2019) or GC methods (Takashi et al., 1989; Jeong et al., 1999; Abe et al., 2000) or by recently developed supercritical fluid chromatography (Khater et al., 2015). Determination of panthenol EE by a spectrometric technique with multivariate calibration (MVC) has not been reported yet. Moreover, this connection offers many advantages compared to traditional chiral methods. The rapidity and simplicity of these methods is appealing and reduces the analytical time and cost of chiral analysis because there is no need of expensive chiral columns and large amount of solvents. Chiral analysis of various drugs by infrared spectrometry with chemometric evaluation of data was performed. Main features of UV/VIS spectrometry with MVG methods can be found in the review by Poláček et al. (2016).

Our research focuses on the feasibility of panthenol EE determination by NIR and UV/VIS spectrometry by MVG methods using $\beta$-cyclodextrin $(\beta-\mathrm{CD})$ as the chiral selector. MVC me- 
thods, principal component regression (PCR) and partial least square method (PLS) were compared for the best identification of panthenol enantiomers from NIR and UV/VIS spectral data. The studied methods were applied to the chiral analysis of panthenol in pharmaceutical preparations, e.g. panthenol tablets and eye drops containing panthenol as an adjuvant.

\section{Materials and methods}

\section{Reagents and methods}

Experiments were performed using chemicals of analytical reagent grade and doubly distilled water. D- and DL-panthenol, native $\beta$-CD, p-dimethylaminobenzaldehyde, acetonylacetone and methanol were purchased from Sigma-Aldrich (United States). Ammonia and sodium carbonate were obtained from Centralchem (Slovakia).

Stock solutions of D and DL-panthenol (45 mmol $\cdot \mathrm{L}^{-1}$ ) for NIR measurements were prepared by dissolving $4.5 \mathrm{~g}$ of each enantiomer in $\beta$-CD solution $\left(45 \mathrm{mmol} \cdot \mathrm{L}^{-1}\right)$. Pure $\beta$-CD solution at the same concentration was used as a blank.

To perform UV/VIS measurements; $100 \mathrm{mg}$ of each panthenol enantiomer was dissolved and made up with water to $250 \mathrm{~mL}$ in a volumetric flask. Then, $2 \mathrm{~mL}$ of these stock solutions were pipetted into $25 \mathrm{~mL}$ volumetric flasks and an appropriate amount of acetonylacetone solution $(1 \mathrm{~mL} / 100 \mathrm{~mL}$ $0.5 \mathrm{~mol} \cdot \mathrm{L}^{-1}$ of sodium carbonate) with $10 \mathrm{~mL}$ of methanol was added. Subsequently, the flask was put into a water-bath at $95-98^{\circ} \mathrm{C}$ for $30 \mathrm{~min}$. Upon cooling, $2 \mathrm{~mL}$ of p-dimethylaminobenzaldehyde solution were added into the flask which was shaken properly. The mixed solutions were made up to the final volume with methanol. After $15 \mathrm{~min}$, appropriate volumes of these solutions were transferred into $10 \mathrm{~mL}$ flasks (total concentration of panthenol was $\left.0.8 \mathrm{mmol} \mathrm{L}^{-1}\right)$ and filled up with $\beta$-CD solution $\left(0.8 \mathrm{mmol} \cdot \mathrm{L}^{-1}\right)$ to the mark. The blank solution was prepared using $2 \mathrm{~mL}$ of distilled water instead of the panthenol solution.

All solutions for each measurement were prepared daily.

\section{Samples}

Pharmaceutical products were purchased from a local pharmacy. Panthenol tablets contain $100 \mathrm{mg}$ of panthenol per tablet and two eye drops consist of $10 \mathrm{mg} \cdot \mathrm{L}^{-1}$ of panthenol in the drops base.

After homogenization of ten tablets, corresponding amounts were weighted and handled according to the above procedures. Aliquot parts of drops' samples were pipetted and processed for both proposed methods as pure substance.

\section{Apparatus}

\section{UV/VIS spectrometry}

VIS absorption spectra from 400 to $700 \mathrm{~nm}$ with $1 \mathrm{~nm}$ resolution were recorded by a UV/VIS spectrometer (Thermo Scientific, USA) equipped with a $1.0 \mathrm{~cm}$ long quartz cell at the room temperature. Scan speed was $200 \mathrm{~nm} \cdot \mathrm{min}^{-1}$. Spectral range between 450 and $650 \mathrm{~nm}$ (201 spectral points) was used to create multivariate calibration models.

\section{NIR spectrometry}

Near-infrared absorption spectra in the range from $4000 \mathrm{~cm}^{-1}$ to $12000 \mathrm{~cm}^{-1}(2500-833 \mathrm{~nm})$ with $2 \mathrm{~cm}^{-1}$ resolution were collected using a spectrophotometer NICOLET 8700TM (Thermo Scientific, USA) equipped with a quartz cell with an optical path of $5 \mathrm{~mm}$. An average of 150 scans was performed to obtain the final spectrum.

\section{Chemometric data analysis}

Spectral data were exported to ASCII data files and calculations were performed by MATLAB 2015b (The MathWorks Inc., USA, 2015) and PLS Toolbox version 8.1 (Eigenvector Research Inc., USA, 2015). EE of panthenol was determined from NIR and UV/VIS spectral data by PCR and PLS MVC methods. In molecular spectrometry, MVC methods are extensively used as they allow for a large number of predictor variables and tolerate correlations between these variables within the measured spectral data (Wise et al., 2007). In the calibration process, a mathematical model has been built up to assign the spectral data to the desired concentration. Each calibration model was tested by a cross-validation method $(\mathrm{CV})$ to estimate the prediction error with the basic idea to divide the available data into two parts: training data and testing data, respectively. Training data are used for fitting the model, while testing data are used for validating the prediction performance of the model. Decomposition of original spectral matrix into a new data matrix in PCR and PLS is realized in a different way. PCR only considers spectral data within the transformation process and generates latent variables from the independent ones while PLS actively sets up a model using two sets of latent variables during the transformation, which involves both spectral and concentration data. The new matrix consists of principal components that are all orthogonal to each other. The first principal component has the highest variance in the spectral data, while the remaining variance is used to find the next principal component, etc. To determine the optimal number of components, the root mean square error of cross-validation (RMSECV) is calcu- 
lated for each model separately. The first model is constructed only from the first component and the next one is created from the progressively growing number of components. The lowest RMSEGV value helps to select an optimal calibration model. The most suitable regression is chosen by assessing the performance of prediction models characteristics based on the root mean square error of the calibration (RMSEC), the root mean square error of crossvalidation (RMSECV), the root mean square error of prediction (RMSEP) and scaled RMSEP against actual value, the root mean square relative error of prediction in percent (RMSE \%RE) (Wise et al., 2007). The PCR method was described in earlier papers (Wise et al., 2007; Okonkwo et al., 2013) and more information on PLS can be found in Wold et al. (2001) and Wise et al. (2007).

MVC calibration models enable describing even small spectral changes and fluctuations in data, originating in various interactions between inclusion complexes formed by enantiomers of panthenol and $\beta$-CD. Data from NIR and UV/VIS spectrometry were used to construct such calibration models.

As for the calibration models samples preparation, 100 samples were prepared by mixing D-panthenol (with the mole fraction from $50.0 \%$ to $100.0 \%$ ) and L-panthenol (in the interval of $0-50 \%$ ). These samples, which form inclusion complexes with $\beta$-CD, have a fixed total panthenol guest concentration and a fixed $\beta$-CD host concentration. The PCR and PLS calibration models were built based on the spectral data obtained from the measurements of 70 samples and 30 samples were used for their cross-validation. No interference in the measured ranges of the proposed methods was observed. After choosing the most suitable models, enantiomeric compositions in pharmaceutical preparations were predicted by both MVC methods. Autoscale pre-processing with leave-one-out crossvalidation was performed for the calibration, prediction, and sample datasets.

\section{Analytical figure of merit}

Net analyte signal (NAS), defined as a part of the measured signal unique for the considered enantiomer, is used for the characterization of MVC model quality. These values are used to estimate the figure of merit in calibration models using equations described in an earlier study (Ferré et al., 2003).

The root mean square percent relative error (RMS \%RE) is a useful figure of merit for quantitatively expressing the predictive efficiency of models (Williams et al., 2006).

\section{Results and discussion}

\section{NIR spectrometry}

Fig. 1 shows the NIR spectra of D-panthenol, panthenol in a racemic mixture, and their inclusion complexes with $\beta$-CD measured in a $5 \mathrm{~mm}$ pathlength cell.

As shown in the NIR spectra in Fig. 1, spectral changes in panthenol enantiomers and their diastereomeric complexes with $\beta$-CD can be observed.

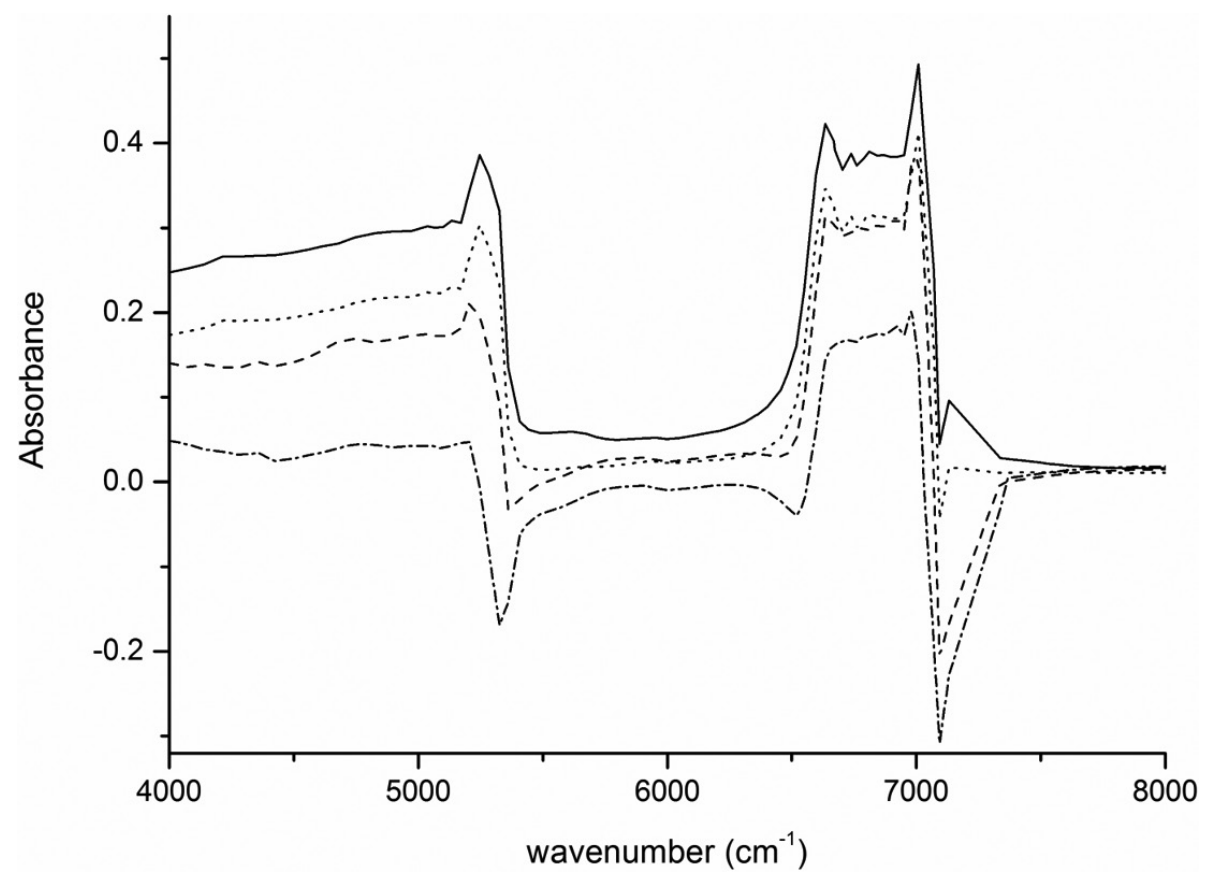

Fig. 1. NIR spectra of panthenol racemic mixture (solid line), D-panthenol (dotted line), complexes of $\beta$-CD with DL-panthenol (dashed line) and dexpanthenol (dash-dotted line). 
The most remarkable differences are observed in the range from 4000 to $8000 \mathrm{~cm}^{-1}$. It is relatively difficult to assign significant bands in the spectra completely and unambiguously as the overtones of $\mathrm{O}-\mathrm{H}$ group and $\mathrm{N}-\mathrm{H}$ group and their combinations may be responsible for several bands at $4500-5000 \mathrm{~cm}^{-1}$ and $6200-7200 \mathrm{~cm}^{-1}$. The $4000-4500 \mathrm{~cm}^{-1}$ and $7000-7200 \mathrm{~cm}^{-1}$ bands can be assigned to combinations of $\mathrm{C}-\mathrm{H}$ group overtones. The most suitable calibration models for the determination of panthenol EE were obtained by PCR and PLS multivariate regression methods. The PCR and PLS models were constructed from the full NIR spectra (4000 experimental points) and three selected wavenumber ranges: the first one at 5200-5400 $\mathrm{cm}^{-1}$ (100 experimental points), the second at 6400-7200 $\mathrm{cm}^{-1}$ (400 experimental points) and the third at $4000-8000 \mathrm{~cm}^{-1}(2000 \mathrm{ex}-$ perimental points). The RMS \% RE value of each model was calculated and used to select the spectral region where the best prediction of $\mathrm{EE}$ is reached. Fig. 2 presents the RMS \%RE values obtained for the EE prediction of D- and L-panthenol from PCR and PLS models. Based on these values, the wavenumber range with maximal recognition of enantiomers was selected. The lowest RMS \% RE values for the enantiomers were obtained in the range of $6400-7200 \mathrm{~cm}^{-1}$ by both regression methods. Also, lower RMS \% RE values indicate proper prediction of EE by PLS models compared to PCR regression in all cases.

Important characteristics of PCR and PLS calibration models (lower number of latent variables, higher sensitivity) and the figures of merit (Tab. 1) confirmed their suitability for EE determina- tion in all selected spectral ranges. PCR and PLS models are more suitable for the prediction of the D-panthenol content than for the L-enantiomer in enantiomeric compositions.

Coefficients of determination of the calibration cross-validation and prediction are above $98 \%$, indicating acceptable prediction of $\mathrm{EE}$ by both calibration models. In case of PLS models, lower accuracy (RMSEs) values were obtained. Moreover, PLS models interpret higher \% of concentration variance compared to PCR models. Likewise, calculated values of sensitivity and analytical sensitivity are in favor of PLS models.

The best PLS models were chosen from NIR spectral data in the range of $6400-7200 \mathrm{~cm}^{-1}$ for the determination of panthenol EE in pharmaceutical products.

\section{UV/VIS spectrometry}

The wavelength of maximum absorbance of panthenol derivatives is at $\lambda_{\max }=525 \mathrm{~nm}$. As it can be seen in Fig. 3, D-panthenol and the racemic mixture in water without $\beta$-CD show very similar absorbance.

However, after addition of $\beta$-CD to the solution, the absorbance of D-panthenol increased. Comparing individual absorbance, formation of inclusion complex of $\mathrm{D}$-panthenol and $\beta$-CD is preferred as the absorbance increases proportionally with higher ratio of D-panthenol in the mixture.

The PCR and PLS calibration models were created from UV/VIS data measured in the wavelength range from 430 to $580 \mathrm{~nm}$ (150 experimental points). Characteristics of the calibration models and the selected figures of merit are presented in Tab. 2.

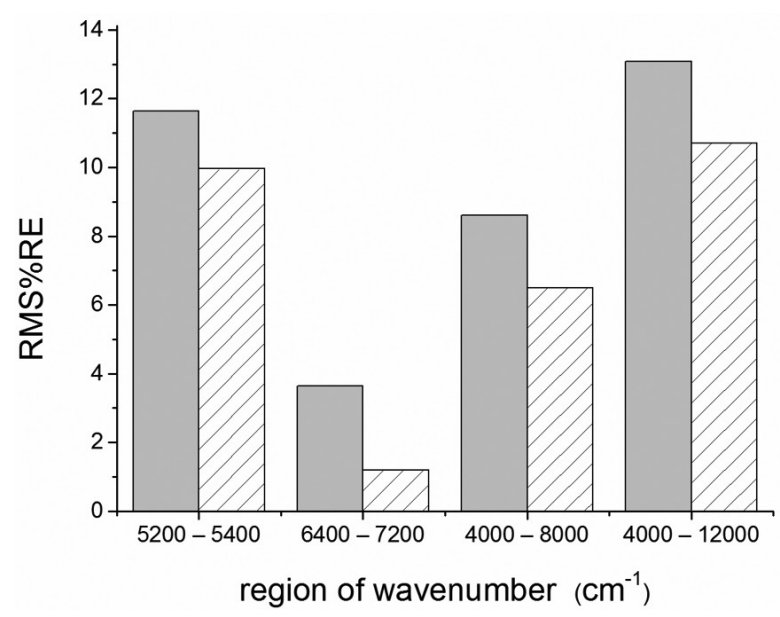

a)

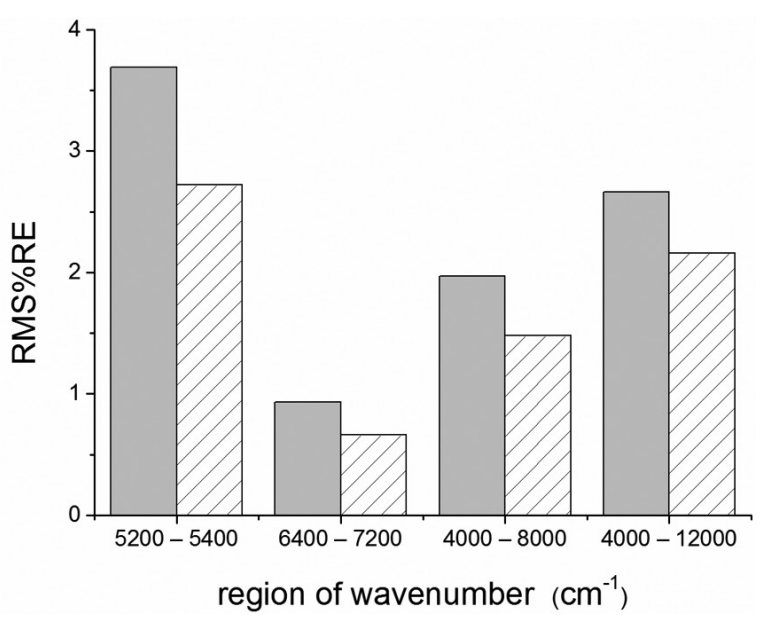

b)

Fig. 2. RMS \% RE values for $\beta$-CD inclusion complexes with panthenol from PCR (A) and PLS (B) calibration models; D-panthenol (light gray), L-panthenol (cross-hatched). 


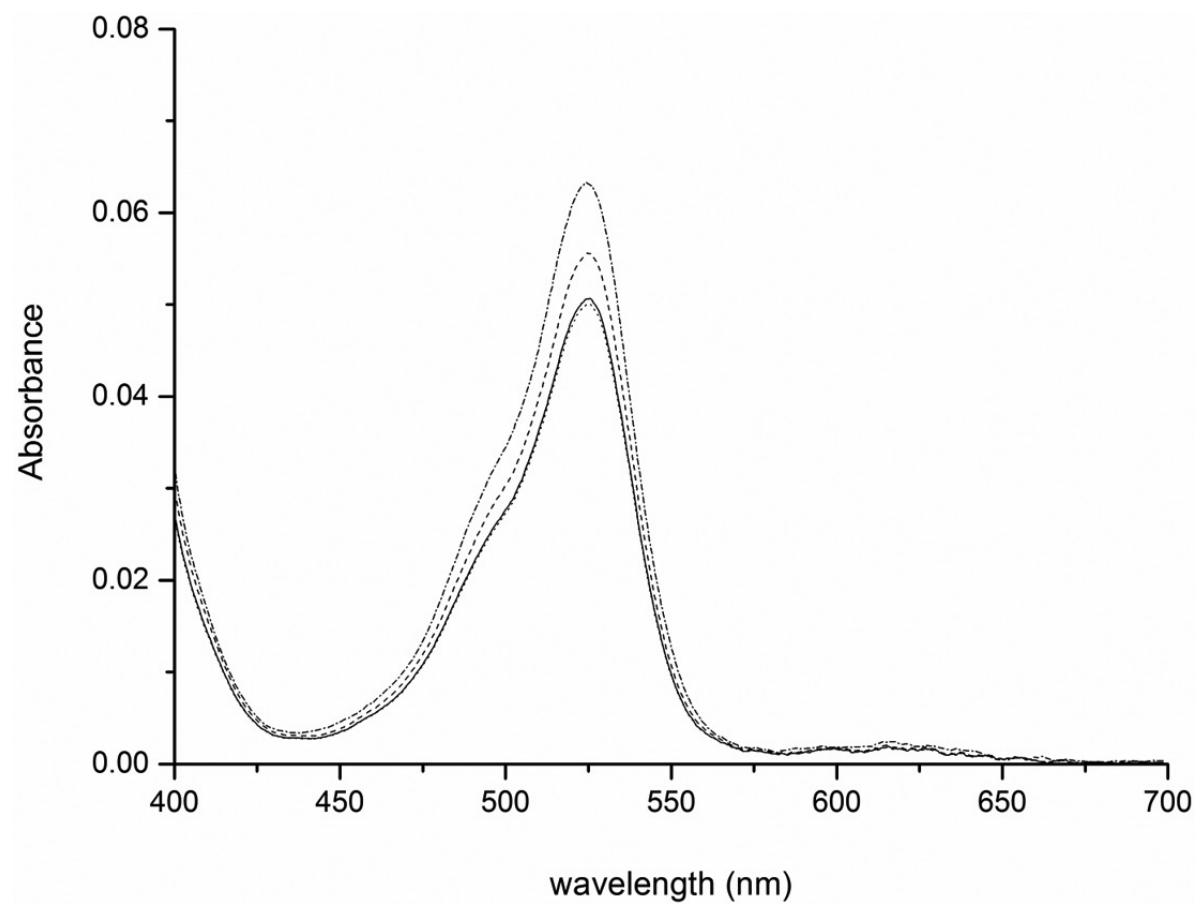

Fig. 3. UV/VIS spectra for panthenol racemic mixture (solid line), D-panthenol (dotted line) in water and inclusion complexes of $\beta$-CD with DL-panthenol (dashed line) and dexpanthenol (dash-dotted line).

The calibration models consist of three latent variables and explain more than $99 \%$ of variance in the spectral data and over $93 \%$ of the concentration variance. As for the PLS models, the coefficients of determination of calibration, cross-validation and prediction are closer to one; all these values are better compared to the PCR model. In addition, better values of the figure of merit were achieved by the PLS model, which were thus selected for UV/VIS spectrometry determination of panthenol EE in pharmaceutical products.

Tab. 1. The figure of merit and predicted values of PCR and PLS calibration models from NIR data for the determination of enantiomeric excess of panthenol.

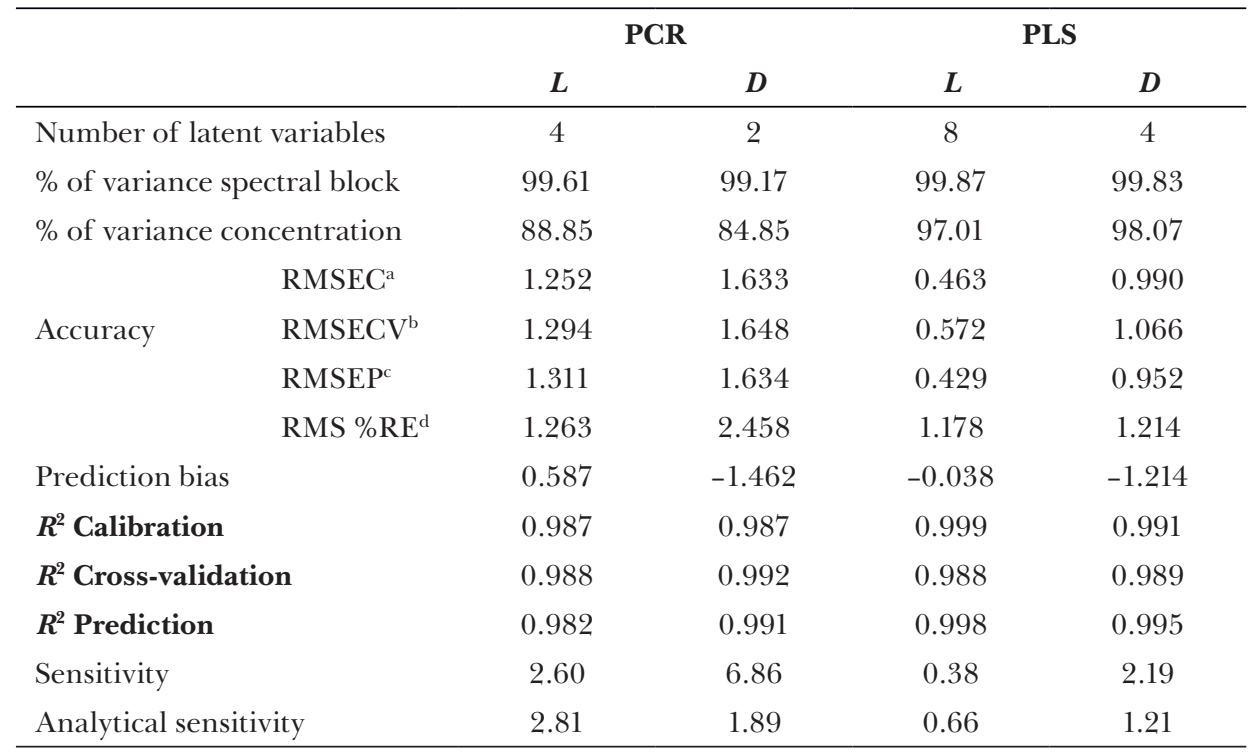

${ }^{\mathrm{a}} \mathrm{RMSEC}$ - root mean square error of calibration,

${ }^{\mathrm{b}} \mathrm{RMSECV}$ - root mean square regression error of cross-validation,

${ }^{\mathrm{C}} \mathrm{RMSEP}$ - root mean square error of prediction,

${ }^{\mathrm{d}} \mathrm{RMS} \% \mathrm{RE}$ - root mean square percent relative error. 
Tab. 2. The figure of merit and predicted values of PCR and PLS calibration models from UV/VIS data for the determination of panthenol enantiomeric excess in synthetic mixtures.

\begin{tabular}{|c|c|c|c|c|c|}
\hline & & \multicolumn{2}{|c|}{ PCR } & \multicolumn{2}{|c|}{ PLS } \\
\hline & & $L$ & $D$ & $L$ & $D$ \\
\hline \multicolumn{2}{|c|}{ Number of latent variables } & 3 & 3 & 3 & 3 \\
\hline \multicolumn{2}{|c|}{$\%$ of variance spectral block } & 99.06 & 99.06 & 99.06 & 99.06 \\
\hline \multicolumn{2}{|c|}{$\%$ of variance concentration } & 97.02 & 93.47 & 97.18 & 94.74 \\
\hline \multirow{4}{*}{ Accuracy } & RMSEC $^{\mathrm{a}}$ & 1.663 & 1.880 & 1.589 & 1.401 \\
\hline & RMSECV $^{\mathrm{b}}$ & 1.591 & 1.754 & 1.584 & 1.462 \\
\hline & RMSEP $^{c}$ & 1.784 & 1.822 & 1.543 & 1.406 \\
\hline & RMS \% RE & 1.781 & 4.484 & 1.657 & 1.882 \\
\hline \multicolumn{2}{|c|}{ Prediction bias } & -1.064 & -1.154 & -1.087 & -1.485 \\
\hline \multicolumn{2}{|c|}{$R^{2}$ Calibration } & 0.970 & 0.945 & 0.982 & 0.985 \\
\hline \multicolumn{2}{|c|}{$R^{2}$ Cross-validation } & 0.949 & 0.911 & 0.977 & 0.987 \\
\hline \multicolumn{2}{|c|}{$R^{2}$ Prediction } & 0.984 & 0.921 & 0.988 & 0.973 \\
\hline \multicolumn{2}{|l|}{ Sensitivity } & 6.45 & 1.48 & 4.27 & 1.13 \\
\hline \multicolumn{2}{|c|}{ Analytical sensitivity } & 3.65 & 0.33 & 2.59 & 0.61 \\
\hline
\end{tabular}

${ }^{a}$ RMSEC - root mean square error of calibration,

${ }^{\mathrm{b}} \mathrm{RMSECV}$ - root mean square regression error of cross-validation,

${ }^{\mathrm{C}} \mathrm{RMSEP}$ - root mean square error of prediction,

${ }^{\mathrm{d}} \mathrm{RMS} \% \mathrm{RE}$ - root mean square percent relative error.

\section{Analysis of pharmaceutical products}

Tab. 3 presents the results of panthenol EE determination in tablets and eye drops of two producers. EE determination was realized by PLS models constructed from spectra acquired by NIR spectrometry in the wavenumber range of $6400-7200 \mathrm{~cm}^{-1}$ and those obtained from UV/VIS data measured in the wavelength range of $430-580 \mathrm{~nm}$. Outcomes calculated by the PLS calibration model from the NIR spectra were in good agreement with those obtained by UV/VIS spectrometry.

Tab. 3. Enantiomeric excess of D-panthenol in pharmaceutical samples.

\begin{tabular}{lcc}
\hline & \multicolumn{2}{c}{ EE of D-panthenol (\%) } \\
samples & NIR-PLS & UV/VIS-PLS \\
\hline tablets & $98.25 \pm 1.32$ & $97.95 \pm 1.01$ \\
eye drops 1 & $95.46 \pm 1.74$ & $96.11 \pm 1.44$ \\
eye drops 2 & $95.71 \pm 1.48$ & $95.99 \pm 1.25$ \\
\hline
\end{tabular}

Pharmaceutical samples, measured three times, were statistically evaluated on a $95 \%$ confidence level $(\alpha=0.05)$ of the Fisher $(F)$ test and two-sample $t$-test; $p$-values of the two-tailed $F$-test fall within the range of $\langle 0.11 ; 0.51\rangle$, indicating no significant differences between the variance values determined by NIR or UV/VIS spectrometry. Since $p$-values of the two-tailed $t$-test are in the interval of $\langle 0.25 ; 0.73\rangle$, it can be concluded that there is no significant dif- ference in the results of the determination of $\mathrm{EE}$ in tablets and eye drops obtained by these spectrometric methods using multivariate PLS calibration.

\section{Conclusion}

In this paper, the possibility of using cheap and rapid methods based on NIR and UV/VIS spectrometry for panthenol EE determination in tablets and eyes drops is outlined. Inclusion complexes of panthenol with $\beta$-CD show small spectral differences and MVC methods, PCR and PLS, have to be used for the quantification of the relationship between the spectral data and the panthenol EE. Validation of the proposed methods was performed exploiting the model characteristics and the figure of merit. The PLS calibration models are preferred to the PCR ones for the determination of panthenol EE. Results of the two spectrometric methods were verified by the Student $t$-test and Fisher $F$-test. Both methods have been shown as promising in terms of the chiral analysis of pharmaceutical products where it is necessary to determine panthenol EE.

\section{Acknowledgement}

This work was supported by the Slovak Research and Development Agency under the contract No. APVV-150455. P.M. is grateful to the HPC center at the STU in Bratislava, which is a part of the Slovak Infrastructure of High Performance Computing (SIVVP project, ITMS 
code 26230120002, funded by the European region development funds, ERDF), for the computational time and resources made available.

\section{References}

Abe I, Terada K, Nakahara T (2000) Biomed. Chromatogr. 14: $125-129$.

Ebner F, Heller A, Rippke F, Tausch I (2002) Am. J. Clin. Dermatol. 3: 427-433.

Ferré J, Faber NM (2003) Chemometr Intell Lab Sys 69: 123-136.

Jeong HJ, Lee MH, Ro KW, Hur CW, Kim JW (1999) Int J. Cosmet. Sci. 21: 41-50.

Hroboňová K, Lomenova A (2019) Chirality 32: 191-199.

Khater S, West C (2015) J. Pharm. Biomed. Anal. 102: $321-325$.
König WA, Sturm U (1985) J. Chromatogr. 328: 357-361.

Mao X, Hu X, Pan W (2010) Chin. J. Chromatogr. 28: $1061-1066$.

Okonkwo EN, Okeke JU, Nwabueze JC (2013) Bayero J. Pure Appl. Sci. 6: 27-31.

Poláček R, Májek P (2016) Chem. Listy 110: 18-25.

Takashi A, Hiroyasu M, Hiroshi O (1989) J. Chromatogr. A 474: 405-410.

Wise BM, Gallagher NB, Bro R, Shaver JM, Windig W, Koch RS (2007) PLS Toolbox 4.0, Eigenvector Research Inc., Wenatchee, USA.

Wold S, Sjostrom M, Eriksson L (2001) Chemometr Intell Lab Sys 58: 109-130.

Xie YM, Luo J, Tang XH, Yang D, Huo XF, Liu A, Hu X, Song X, Song H (2009) Chromatographia 69: $1025-1029$. 